\title{
Typing of Pseudomonas aeruginosa from hospitalized patients: a comparison of susceptibility and biochemical profiles with genotype
}

A.L. Freitas ${ }^{1,2}$ and A.L. Barth ${ }^{1,3}$
Correspondence

A.L. Freitas

Barros Cassal, 666/806

90035-030 Porto Alegre, RS

Brasil

Fax: + 51-3316-8310

E-mail: usha@via-rs.net

Received January 10, 2003 Accepted October 14, 2003
${ }^{1}$ Faculdade de Farmácia, Universidade Federal do Rio Grande do Sul, Porto Alegre, RS, Brasil

${ }^{2}$ Escola de Farmácia, Universidade Católica de Pelotas, Pelotas, RS, Brasil ${ }^{3}$ Unidade de Pesquisa Biomédica, Serviço de Patologia Clínica,

Hospital de Clínicas de Porto Alegre, Porto Alegre, RS, Brasil

\begin{abstract}
Typing techniques are essential for understanding hospital epidemiology, permitting the elucidation of the source of infection and routes of bacterial transmission. Although DNA-based techniques are the "gold standard" for the epidemiological study of Pseudomonas aeruginosa, antibiotic profiles and biochemical results are used because they are easy to perform and to interpret and relatively inexpensive. Antibiotypes (susceptibility profiles) and biotypes (biochemical profiles) were compared to genotypes established by DNA restriction enzyme analysis in 81 clinical isolates of $P$. aeruginosa from three hospitals in Porto Alegre, Brazil. The epidemiological relationship among patients was also evaluated. Susceptibility and restriction profiles were discrepant in more than $50 \%$ of the cases, and many antibiotypes were observed among isolates from the same genotype. Furthermore, susceptibility profiles did not allow the distinction of isolates from unrelated genotypes. Since a large number of isolates (63\%) yielded the same biochemical results, only 10 biotypes were detected, showing that this typing method has a low discriminatory power. On the other hand, DNA restriction enzyme typing allowed us to establish 71 distinct types. Epidemiological data about the relation among $P$. aeruginosa isolates were not conclusive. The results of the present study indicate that the only method that can establish a clonal relation is DNA restriction enzyme typing, whereas the other methods may cause misleading interpretations and are inadequate to guide proper infection control measures.
\end{abstract}

\section{Introduction}

Despite the advances in hospital care and the introduction of a wide variety of antimicrobial agents, Pseudomonas aeruginosa continues to be a common cause of nosoco-
Key words

- Pseudomonas aeruginosa

- Typing

- Genotyping

- Phenotypic markers mial infections. Typing techniques are essential to establish a clonal relationship between individual isolates in the hospital setting, to recognize outbreaks, and to elucidate the source of infection (1). Although DNAbased techniques have been successfully 
applied to the epidemiological study of $P$. aeruginosa (2-4), these procedures are time consuming and expensive and require specific equipment $(5,6)$. Thus, antibiotic susceptibility and biochemical tests are usually employed routinely by clinical laboratories.

Although susceptibility tests provide profiles that are used in infection control, in most cases these data cannot confirm clusters of nosocomial cross-infection or distinguish unrelated infections (5,7-9), especially when multiresistant strains are analyzed $(6,10)$. However, quantitative analysis of antibiograms by comparing disk zone sizes has been considered useful for nosocomial infection control in a few situations (11).

Biochemical tests in combination with production of pigment and hemolysis allow the determination of distinct biotypes. $P$. aeruginosa displays a uniform response to some biochemical tests which are used for identification purposes. However, the variability of the response to other tests can provide different profiles (biotype) that are useful to distinguish clinical isolates. The carbohydrates used for typing include galactose, mannose, mannitol and rhamnose, with utilization rates of 22 to $81 \%$ (12). $P$. aeruginosa metabolizes carbohydrates by the oxidative pathway and requires a special culture medium to be detected. Degradation of carbohydrates is evaluated by the oxidative-fermentative (OF) medium. However, the small production of acid by oxidation can be neutralized by alkali released from the breakdown of peptone, preventing the detection of sugar utilization. Therefore, utilization may be better demonstrated in ammonium salt medium, mainly because of the lack of peptone in the medium (13). Urea hydrolysis, which is positive for $43 \%$ of $P$. aeruginosa strains, and hemolysis on blood agar, occurring in about half of the clinical isolates, are other useful biochemical tests (13). Most $P$. aeruginosa strains produce pyocyanin and fluorescein, which gives the characteristic blue-green color to colonies in agar cultures. Some P. aeruginosa strains can produce colonies with distinct color due to other pigments, or even non-pigmented colonies (14).

In the present report, 81 clinical isolates of $P$. aeruginosa from hospitalized patients were evaluated for epidemiological relatedness using two phenotypic methods (antibiotic susceptibility and biochemical patterns), molecular analysis (restriction analysis of DNA followed by pulsed-field gel electrophoresis) and epidemiological information (date and place of isolation). Phenotypic and genotypic data were compared to determine the accuracy of antibiotic profiles and biochemical results were compared to establish a clonal relationship among clinical isolates of $P$. aeruginosa.

\section{Material and Methods}

Clinical isolates of $P$. aeruginosa recovered from patients with non-cystic fibrosis admitted to three hospitals in Porto Alegre, Brazil, were analyzed in this study. P. aeruginosa was identified on the basis of production of pigment, oxidase, glucose, arginine, and nitrate, and growth on cetrimide agar (12).

The susceptibility tests were performed by the agar disk diffusion method according to the guidelines of the National Committee for Clinical Laboratory Standards (15). To represent the different classes of antimicrobial agents commonly used for the treatment of $P$. aeruginosa we used amikacin, aztreonam, carbenicillin, ceftazidime, ciprofloxacin, imipenem and ticarcillin-clavulanic acid (BBL-Becton Dickinson Microbiology, Cockeysville, Franklin Lakes, NJ, USA). Differences in the results from susceptibility to resistance and from resistance to susceptibility obtained with at least one of the agents characterized a different antibiotype, represented by a lower case letter. Differences in the results from susceptibility to intermedi- 
ate, or the contrary, characterized a subtype, represented by an Arabic number added to the antibiotype letter.

Biotyping was performed by observation of hemolysis after growth for $24 \mathrm{~h}$ under transmitted light. Hemolytic isolates were divided into two groups according to observation of $\alpha$ or $\beta$ hemolysis. Production of pigment was determined in Mueller-Hinton medium incubated for $24 \mathrm{~h}$ at $35^{\circ} \mathrm{C}$, and pigmented colonies were classified according to their color.

Glucose utilization was first assessed with OF medium (BBL-Becton Dickinson Microbiology) using two tubes, one of them overlaid with paraffin oil. Carbohydrate degradation was performed using one tube containing OF medium and one containing ammonium salt sugar medium supplemented with $1 \%$ galactose, mannose, mannitol or rhamnose. The tubes were incubated up to 4 days before a negative result was considered. Urea hydrolysis was performed by the method of Christensen (for a review, see Ref. 12). Each group of identical results for pigment, hemolysis, urea hydrolysis and carbohydrate utilization was considered to be a biotype and coded with a number.

Epidemiological relatedness was estimated by geographic and temporal links among patients from each hospital. Patients were considered to be related when they were treated at the same unit and $P$. aeruginosa was isolated within five days. A relation number $(\mathrm{R})$ was given to each group of related patients.

Restriction enzyme analysis of DNA followed by pulsed-field gel electrophoresis was performed as described previously (16). Briefly, the bacteria were embedded in agarose blocks and digested with the restriction endonuclease SpeI (Gibco BRL Life Technologies, Rockville, MD, USA). Electrophoresis was carried out using a CHEF DR II apparatus (Bio-Rad, Richmond, CA, USA) at $14^{\circ} \mathrm{C}, 5.9 \mathrm{~V} / \mathrm{cm}$, for $22 \mathrm{~h}$, with a time switch of 5 to $50 \mathrm{~s}$. The lambda ladder (48.5 kb; Sigma, St. Louis, MO, USA) was used as the molecular weight marker. The gels were stained with ethidium bromide and the image was acquired with a Chemilmager 4000 (Tech Corporation, Minneapolis, MN, USA). Restriction fragment profiles were compared visually and the criteria of Tenover et al. (17) were used to establish relationships among the isolates. Restriction enzyme analysis was considered to be the "gold standard" for these comparisons $(2,3)$.

\section{Results}

We analyzed 81 clinical isolates obtained from patients admitted to three different hospitals between September 1998 and May 1999. Fifty-four percent of the isolates were obtained from the respiratory tract, $28 \%$ from urine, $6.5 \%$ from blood, and $11.5 \%$ from a variety of other sources (catheters, abdominal secretions, skin).

The susceptibility results allowed us to establish 26 major antibiotypes, 3 of them including subtypes, while unique profiles for the isolate were observed in 18 cases. A total of $23(28.4 \%)$ isolates were susceptible to all agents tested, but only 3 were multiresistant (Table 1). Isolates were considered to be multiresistant when they displayed in $v i$ tro resistance to all tested agents.

\begin{tabular}{|c|c|c|c|c|c|c|c|c|c|}
\hline Antibiotype & $N$ & $\%$ & AMI & ATM & CAR & CAZ & CIP & IMI & $\mathrm{TIC}$ \\
\hline a & 23 & 28.4 & $S$ & $S$ & $S$ & $S$ & $S$ & $S$ & $S$ \\
\hline b & 3 & 7.7 & $\mathrm{R}$ & $R$ & $\mathrm{R}$ & $R$ & $\mathrm{R}$ & $\mathrm{R}$ & $\mathrm{R}$ \\
\hline$C^{*}$ & 9 & 11.1 & $\mathrm{R}$ & $S$ & $\mathrm{R}$ & $S$ & $\mathrm{R}$ & $S$ & $\mathrm{R}$ \\
\hline$d^{*}$ & 9 & 11.1 & $S$ & $S$ & I & $S$ & $S$ & $S$ & S \\
\hline$e^{*}$ & 9 & 11.1 & $S$ & $S$ & $\mathrm{R}$ & $S$ & $S$ & $S$ & $S$ \\
\hline$f$ & 3 & 7.7 & $S$ & I & $\mathrm{R}$ & $S$ & $S$ & $S$ & $\mathrm{R}$ \\
\hline g & 3 & 7.7 & $\mathrm{R}$ & $S$ & $\mathrm{R}$ & $S$ & $\mathrm{R}$ & $S$ & $S$ \\
\hline h & 2 & 7.7 & 1 & $S$ & $\mathrm{R}$ & $S$ & $\mathrm{R}$ & $S$ & $\mathrm{R}$ \\
\hline k & 2 & 2.5 & $\mathrm{R}$ & I & $\mathrm{R}$ & S & $\mathrm{R}$ & $\mathrm{R}$ & $\mathrm{R}$ \\
\hline
\end{tabular}


Production of pigment was observed in 79 of 81 isolates, most of them $(82.7 \%)$ displaying the characteristic green color. All isolates were hemolytic and most of them demonstrated $\beta$ hemolysis $(91.3 \%)$. The results of carbohydrate utilization were the same for both the OF and ammonium salt sugar media, although the time needed to

Table 2. Characteristics of 81 Pseudomonas aeruginosa isolates from patients with an epidemiological relation.

\begin{tabular}{lcccc}
\hline $\begin{array}{l}\text { Epidemiological } \\
\text { relation }\end{array}$ & $\begin{array}{c}\text { No. } \\
\text { of isolates }\end{array}$ & $\begin{array}{c}\text { No. of } \\
\text { antibiotypes }\end{array}$ & $\begin{array}{c}\text { No. of } \\
\text { biotypes }\end{array}$ & $\begin{array}{c}\text { No. of } \\
\text { PFGE patterns }\end{array}$ \\
\hline R1 & 9 & 7 & 5 & 7 \\
R2 & 4 & 3 & 2 & 4 \\
R3 & 4 & 3 & 1 & 4 \\
R4 & 4 & 4 & 4 & 3 \\
R5 & 3 & 3 & 2 & 3 \\
R6 & 3 & 3 & 1 & 3 \\
R7 & 3 & 3 & 1 & 3 \\
R8 & 3 & 1 & 1 & 3 \\
R9 & 2 & 2 & 1 & 2 \\
R10 & 2 & 2 & 1 & 2 \\
R11 & 2 & 2 & 1 & 2 \\
R12 & 2 & 1 & 1 & 1 \\
R13 & 2 & 2 & 1 & 2 \\
\hline
\end{tabular}

Epidemiological relatedness was estimated by geographic and temporal links among patients from each hospital. For details, see Material and Methods. Data are reported as number of distinct profiles observed in the group. PFGE = pulsed-field gel electrophoresis (DNA restriction enzyme typing).

Table 3. Characteristics of Pseudomonas aeruginosa with DNA restriction enzyme profiles observed in more than one isolate.

\begin{tabular}{lccl}
\hline Genotype & Antibiotype & Biotype & Epidemiological relation \\
\hline A & $m$ & 1 & no relation \\
A & $\mathrm{p}^{*}$ & 1 & no relation \\
B & $\mathrm{b}$ & 1 & R12 \\
B1 & $\mathrm{b}$ & 1 & no relation \\
B1 & $\mathrm{b}$ & 1 & R12 \\
C & $\mathrm{k}$ & 1 & no relation \\
C1 & $\mathrm{r}^{*}$ & 1 & no relation \\
D & $\mathrm{s}^{*}$ & 6 & R1 \\
D & $\mathrm{d} 1$ & 9 & R1 \\
D & $\mathrm{f} 1$ & 1 & R1 \\
D & $\mathrm{t}^{*}$ & 7 & R4 \\
D & $\mathrm{m} 1$ & 5 & R4 \\
E & $\mathrm{q}^{*}$ & 1 & no relation \\
E & $\mathrm{c} 1$ & 1 & no relation \\
\hline
\end{tabular}

Asterisks indicate that antibiotypes are unique for the isolate. obtain positive results was not always the same. Most isolates were positive for galactose $(99 \%)$, and only a small number metabolized rhamnose (1\%). The results of these tests provided 10 distinct biotypes. Isolates that used urea, galactose, mannose and mannitol and displayed a green pigment and $\beta$ hemolysis belonged to the more frequent biotype (63\% of the cases).

Epidemiological analysis yielded 13 groups that included a variable number of patients. Only 2 of these groups (R8, R12) displayed the same antibiotype (Table 2); however, the same biotype was observed in isolates from patients of 9 groups.

A total of 81 isolates of $P$. aeruginosa were submitted to DNA restriction enzyme molecular typing and 71 different DNA profiles (genotypes) were observed among them. Five genotypes were detected in more than one isolate, and genotype D was observed in 5 isolates (Table 3 ).

Taking the pulsed-field gel electrophoresis profiles as standard, we observed that the same genotype included several distinct antibiotypes, with the exception of multiresistant isolates from clone $\mathrm{B}$ which displayed the same profile in all methods used. Genotype D included five isolates belonging to distinct antibiotypes and biotypes (Table 3 ). However, this profile was also observed in two other unrelated isolates. It was not possible to detect the relationship between the antibiotype and the biotype of the isolates from this clone (Table 3 ).

\section{Discussion}

DNA restriction enzyme analysis (molecular typing) demonstrated that most $P$. aeruginosa isolates belonged to distinct genotypes, demonstrating again that this method had a higher discriminatory power than the phenotypic methods (antibiotyping and biotyping).

The susceptibility typing classified many isolates as antibiotype "a" (fully susceptible to 
antibiotics); however, all of them displayed distinct DNA restriction enzyme profiles. In fact, antibiotype "b" (multiresistant to antibiotics) was the only group that included isolates belonging to the same genotype. This may be due to the difference in the number of isolates classified into these groups, only 3 isolates in antibiotype "b" and 23 isolates in antibiotype "a", and therefore a higher variability of genotypes would be more likely to be found in the latter antibiotype.

Although isolates with unique susceptibility profiles displayed distinct genotypes, the difference in antibiotype may not guarantee clonal distinction, as many isolates of the same genotype displayed distinct susceptibility profiles, revealing the inadequacy of antibiotyping for typing purposes, as previously described $(1,5,9)$.

The inadequacy of antibiotype to confirm a clonal relation among multiresistant isolates has been reported $(6,10)$. However, we found antibiotyping useful since all the multiresistant isolates of this study were included in the same genotype. The low prevalence of multiresistant $P$. aeruginosa observed in our study (2.4\%) may be the factor responsible for the clonal identity of the isolates.

The low discriminatory power of susceptibility tests is not surprising since the power of a method is determined by the number of types defined by it and the relative frequencies of these types (18). Although we obtained a satisfactory number of groups (26 major groups), the heterogeneous distribution of the isolates in the groups $(28.4 \%$ in the same antibiotype) indicated the low discriminatory power of this method.

According to Kiska and Gilligan (12), the rates of utilization of galactose, mannose, mannitol and rhamnose by P. aeruginosa are $81,79,68$ and $22 \%$, respectively, indicating that these tests may be useful to distinguish among clinical isolates. However, in the present study we observed a more homogeneous utilization of carbohydrates than reported by Kiska and Gilligan (12) and, on the basis of this result, we classified most $P$. aeruginosa into the same group, a fact indicating the poor discriminatory power of biotyping.

Phenotypic methods have been used to screen isolates which may be further typed by a more discriminatory test $(7,11,19,20)$, but in the present study we found biotype and antibiotype to be unsatisfactory for screening or typing. The combination of the two methods was also insufficient to establish a distinction among isolates from different genotypes. In fact, failure to obtain good quality data using phenotypic methods has been reported previously, also with respect to isolates from cystic fibrosis patients $(1,5,6$, $9,10,20,21)$.

The isolation of the same bacteria from patients in the same unit may be of help to detect an outbreak (22). However, data from our study and others $(23,24)$ indicate that epidemiological relation among patients is not adequate to establish cross-transmission or outbreaks since no clonal relation may be observed among $P$. aeruginosa strains from these patients. We also observed that isolates of the same genotype may be obtained from unrelated patients, a fact explained by the environmental contribution as a source of infection (25). Phenotypic methods (serotyping and pyocin typing) and pulsed-field gel electrophoresis can demonstrate the same number of identical strains among isolates from an outbreak, while antibiogram profile differs significantly (26).

Although DNA restriction enzyme typing is the "gold standard" for P. aeruginosa typing, because of the time required for determination, epidemiological and phenotypic data, mainly susceptibility profiles, are usually employed to indicate a possible outbreak. However, the present study showed that these data are not acceptable as a presumption of relatedness or distinction among $P$. aeruginosa isolates, confirming that only DNA typing can establish a clonal relation. 


\section{References}

1. Corona ALN, Miranda MGN, Leanos BM, Portilo LG, Hernadez AC, Anthor JR \& Aguilar SB (2001). Epidemiologic study of Pseudomonas aeruginosa in critical patients and reservoirs. Archives of Medical Research, 32: 238-242.

2. Struelens MJ, Schwan V, Deplano A \& Baran D (1993). Genome macro restriction analysis of diversity and variability of Pseudomonas aeruginosa strains infecting cystic fibrosis patients. Journal of Clinical Microbiology, 31: 2320-2326.

3. Grundmann H, Schneider C, Hartung D, Daschner F \& Pitt TL (1995). Discriminatory power of three DNA-based typing techniques for Pseudomonas aeruginosa. Journal of Clinical Microbiology, 33: 528534.

4. Speijer H, Savelkoul PH, Bonten MJ, Stobberingh EE \& Tjhie HT (1999). Application of different genotyping methods for Pseudomonas aeruginosa in a setting of endemicity in an intensive care unit. Journal of Clinical Microbiology, 37: 3654-3661.

5. Williams $T$ (1997). Evaluation of antimicrobial sensitivity patterns as markers of Pseudomonas aeruginosa cross-infection at cystic fibrosis clinics. British Journal of Biomedical Science, 54: 181-185.

6. Rodriguez B, Vasquez E, Sussmann O, Mattar S \& Jaramillo CA (2000). Antimicrobial susceptibility and serotyping of Pseudomonas aeruginosa strains isolated in Bogota, Colombia. European Journal of Clinical Microbiology and Infectious Diseases, 19: 152-154.

7. Bergmans D, Bonten M, van Tiel F, Gaillard C, London N, van der Geest S, Leeuw P \& Stobberingh E (1997). Value of phenotyping methods as an initial screening of Pseudomonas aeruginosa in epidemiologic studies. Infection, 25: 350-354.

8. Traub WH, Scheidhauer R, Leonhard B \& Bauer D (1998). Surveillance of Pseudomonas aeruginosa in intensive care units: clusters of nosocomial cross-infection and encounter of a multiple-antibiotic resistant strain. Chemotherapy, 44: 243-259.

9. Muller MP \& Gubina M (2000). Serotype, antimicrobial susceptibility and clone distribution of Pseudomonas aeruginosa in a university hospital. Zentralblatt für Bakteriologie, 289: 857-867.

10. Mifsud AJ, Watine J, Picard B, Charet JC, Solignac CB \& Pitt TL (1997). Epidemiologically related and unrelated strains of Pseudomonas aeruginosa serotype $\mathrm{O} 12$ cannot be distinguished by phenotypic and genotypic typing. Journal of Hospital Infection, 36: 105116.

11. Giacca M \& Monti CB (1987). Multivariate analysis of antibiograms for typing Pseudomonas aeruginosa. European Journal of Clinical Microbiology, 6: 552-558.

12. Kiska DL \& Gilligan PH (1999). Pseudomonas and Bulkholderia. In: Murray PR, Baron EJ, Pfaller MA, Tenover FC \& Yolken RH (Editors), Manual of Clinical Microbiology. 7th edn. American Society for Microbiology, Washington, DC, USA.

13. Cowan ST (1974). Manual for the Identification of Medical Bacteria. 2nd edn. Cambridge University Press, London, UK.
14. Pitt TL (1988). Epidemiological typing of Pseudomonas aeruginosa. European Journal of Clinical Microbiology and Infectious Diseases, 7: 238-247.

15. NCCLS - National Committee for Clinical Laboratory Standards (1999). Performance Standards for Antimicrobial Disk Susceptibility Tests. Wayne, PA, USA.

16. Kaufmann ME (1998). Pulsed-field gel electrophoresis. In: Woddford $\mathrm{N}$ \& Johnson AP (Editors), Molecular Bacteriology. Protocols and Clinical Applications. Humana Press Inc., Totowa, NJ, USA, 33-50.

17. Tenover F, Arbeit R, Goering R, Mickelsen P, Murray B, Persing D \& Swaminathan B (1995). Interpreting chromosomal DNA restriction patterns produced by pulsed-field gel electrophoresis: criteria for bacterial strain typing. Journal of Clinical Microbiology, 33: 22332239.

18. Hunter PR \& Gaston MM (1989). Numerical index of the discriminatory ability of typing systems: an application of Simpson's index of diversity. Journal of Clinical Microbiology, 26: 2465-2466.

19. Renders N, van Belkun A, Barth A, Goessens W, Mouton J \& Verbrugh $H$ (1996). Typing Pseudomonas aeruginosa strains from patients with cystic fibrosis: phenotyping versus genotyping. Clinical Microbiology and Infection, 1: 261-265.

20. Kinoshita M, Sawabe E \& Okamura N (1997). Concept of segmentation in nosocomial epidemiology: epidemiological relation among antimicrobial-resistant isolates of Pseudomonas aeruginosa. Journal of Infection, 35: 269-276.

21. Silbert S, Barth AL \& Sader HS (2001). Heterogeneity of Pseudomonas aeruginosa in Brazilian cystic fibrosis patients. Journal of Clinical Microbiology, 39: 3976-3981.

22. Bennett JV \& Brachman PS (1992). Hospital Infections. 3rd edn. Little Brown and Company, Boston, MA, USA.

23. Verweij PE, Bijl D, Melchers WJ, Pauw BE, Meis JF, Hoogkamp JA \& Voss A (1997). Pseudo-outbreak of multiresistant Pseudomonas aeruginosa in a hematology unit. Infection Control and Hospital Epidemiology, 18: 128-131.

24. Slama KB, Boudabous A, Skande G, Cherif A, Chedlya F, Boelenst H, Maaskant J, Verbrugh H \& Belkum A (2001). Heterogeneity among infecting strains of $P$. aeruginosa in diverse departments of a large Tunisian hospital. Journal of Hospital Infection, 47: 325-327.

25. Griffith SJ, Nathan C, Selander RK, Chamberlin W, Gordon S, Kabins $S$ \& Weinstein R (1989). The epidemiology of Pseudomonas aeruginosa in oncology patients in a general hospital. Journal of Infectious Diseases, 160: 1030-1036.

26. Sader HS, Pignatari AC, Leme IL, Burattini MN, Tancresi R, Hollis RJ \& Jones RN (1993). Epidemiologic typing of multiply drug-resistant Pseudomonas aeruginosa isolated from an outbreak in an intensive care unit. Diagnostic Microbiology and Infectious Disease, 17: 1318. 